\title{
A Dual Heuristic for Mixed Integer Programming
}

\author{
Yaxian Li $^{\mathrm{a}}$, Ozlem Ergun ${ }^{\mathrm{b}}$, George L. Nemhauser ${ }^{\mathrm{a}}$ \\ ${ }^{a}$ Georgia Institute of Technology \\ ${ }^{b}$ Northeastern University
}

\begin{abstract}
In linear programming based branch-and-bound algorithms, many heuristics have been developed to improve primal solutions, but on the dual side we rely solely on cutting planes to improve dual bounds. We design a dual heuristic which incorporates relaxation algorithms within a branch-and-bound framework to improve dual bounds. We study the effect of solving various relaxations with dual heuristics by conducting a series of computational tests on the multi-dimensional knapsack problem.
\end{abstract}

Keywords: Dual Heuristic, Branch-and-bound, Knapsack, Relaxations

\section{Introduction}

Many primal heuristics have been developed to obtain highquality feasible solutions to mixed integer programming (MIP) problems. For example, neighborhood search iteratively improves the current incumbent solution and diving involves iterative rounding and variable fixing to construct feasible solutions from linear programming (LP) relaxation solutions, see Lodi [1]. Furthermore, primal heuristics such as the feasibility pump developed by Fischetti, Glover and Lodi [2], local branching by Fischetti and Lodi [3], and RINS by Dana, Rothberg and Pape [4] have been implemented successfully within state-of-the-art MIP solvers. However, the only method that is used within MIP solvers to obtain lower bounds for minimization problems is based on solving LP relaxations possibly tightened by the addition of cutting planes. In this paper, we consider using other relaxation algorithms routinely to obtain lower bounds within the Branch-and-Bound(B\&B) algorithm.

Generally, lower bounds can be obtained by solving a relaxation, which is achieved by either enlarging the feasible region, decreasing the value of the objective function over the feasible region or both. All commercial MIP solvers use LP relaxations to obtain lower bounds with cutting planes possibly added to improve lower bounds. While cutting planes can be very effective, after adding a large number of them, the LP relaxations can become more difficult to solve. Another type of relaxation is to keep the integrality constraints and relax complicating constraints by simply dropping, dualizing, or aggregating them, which are called constraint, lagrangian and surrogate relaxations, respectively. The goal is usually to rapidly solve one or a sequence of relaxed problems that are computationally easier to handle.

We denote the constraints that are dropped, dualized or aggregated as relaxed and the rest of the constraints as present in a relaxation problem. Although larger sets of present-constraints in a relaxation lead to better lower bounds, there is a tradeoff between the number of present-constraints and the ease of solving the relaxation. A large number of present-constraints may lead to relaxations that are harder to solve, while a small number may give poor lower bounds. Therefore, our first goal is to understand the impact of the number of present-constraints on lower bounds obtained from different relaxations.

On the other hand, the lower bounds from relaxations with different sets of present-constraints may vary drastically even for a fixed number of present-constraints. In this paper, we show that for certain MIP problems with a large number of constraints, using linear relaxation dual variable information to choose present-constraints is effective in obtaining good lower bounds.

In addition, the quality of the lower bounds obtained with different relaxation techniques may vary depending on the structure of the problem. We compare the lower bounds obtained from constraint and surrogate relaxations. Furthermore, we develop a lazy relaxation. A relaxed-constraint is called lazy if it is allowed to be added back to the formulation when it is found to be violated by a primal feasible solution during the branch and bound algorithm. In this case the lazy-constraint is added back to the present-constraint set. Lazy-constraints can be useful for problems with a huge number of constraints especially when there are constraints that are unlikely to be violated by feasible solutions. When using CPLEX 12.2 as the MIP solver with the lazy-constraints option turned on, the lazy-constraints are only checked each time an integer solution is found.

The constraint and surrogate relaxations relax a fixed set of constraints, which can be a barrier for obtaining good lower bounds if the present-constraints are not chosen properly. The lazy relaxation has the flexibility to modify present-constraints during the B\&B algorithm, while still relying on choosing an initial set of present-constraints to perform well.

We develop a new heuristic algorithm that solves relaxations with possibly different sets of present-constraints. This idea is analogous to a class of neighborhood search based heuristic algorithms, where neighborhoods of feasible solutions are explored by fixing some variables to obtain better solutions, while our algorithm relaxes some constraints and solves relaxations to 
obtain lower bounds. Since fixing variables in a primal problem corresponds to relaxing constraints in its dual problem, we call the scheme of heuristically relaxing constraints a dual heuristic algorithm. The dual heuristic algorithm chooses presentconstraints using information obtained from linear relaxations of the B\&B tree nodes. Thus relaxations with different sets of present-constraints can be explored. Insofar as we know, this is the first heuristic algorithm that uses a neighborhood search idea in the dual space.

We conduct our experiments on the multidimensional knapsack problem MKP, which is a generalization of the knapsack problem with multiple knapsack constraints. For many MKP instances with a large number of constraints, it is difficult to close the relative optimality gaps even with state-of-theart MIP solvers. A main obstacle for efficiently solving MKP is the lack of structure. Since all MKP constraints are knapsack constraints, it is difficult to determine effective relaxations in order to obtain good lower bounds. For MKPs with hundreds or thousands of constraints, adding many cutting planes may cause computational difficulties with branch-and-cut algorithms. These characteristics of large size MKPs call for the development of general-purpose lower-bound-improving strategies, which is the focus of this paper.

The rest of the paper is organized as follows. In Section 2, we introduce the dual heuristic algorithm. In Section 3, we describe the generation of MKP instances for computational experiments. In Section 4, we study strategies for selecting present-constraints in relaxation algorithms. In Section 5, we compare lower bounds obtained from various relaxation algorithms. In Section 6, we give computational results of our dual heuristic algorithm. Section 7 gives conclusions and future research. All of the computational tests are done through CPLEX 12.2 Concert Technology on Linux machines with Dual Xeon E5520 quad-core processor at $2.27 \mathrm{GHz}$ with $48 \mathrm{~GB}$ of memory.

\section{A Dual Heuristic Algorithm}

The MKP can be stated as:

$$
\begin{aligned}
\min c x & \\
\text { s.t. } A x & \geq b \\
x & \geq 0 \text { integer, }
\end{aligned}
$$

with $A \in \mathbb{Z}_{+}^{m \times n}, b \in \mathbb{Z}_{+}^{m}$ and $c \in \mathbb{R}_{+}^{n}$. Let $I P(v)$ be the integer program at a node $v$ of the B\&B tree for problem MKP derived from (1) by adding bound constraints on branching variables and possible cutting planes. In a standard B\&B algorithm, the lower bound for node $v$ is obtained by solving the linear relaxation of $\operatorname{IP}(v)$.

The dual heuristic algorithm solves an additional relaxation while examining a node during the B\&B algorithm. Suppose the constraints $A x \geq b$ are partitioned as $A_{1} x \geq b_{1}$ and $A_{2} x \geq b_{2}$ where $A_{1} \in \mathbb{Z}_{+}^{m_{1} \times n}, A_{2} \in \mathbb{Z}_{+}^{m_{2} \times n}$ and $m_{1}+m_{2}=m$. In the dual heuristic algorithm, we relax constraints $A_{2} x \geq b_{2}$, making them the relaxed constraints, and then solve an IP with fewer constraints. We denote a node as processed if it is pruned or two children nodes are generated from it in the B\&B algorithm and denoted as special if an additional relaxation is solved while processing it.

Let $l b_{\text {Relax }}$ be the lower bound obtained from solving the additional relaxation at a special-node, $l b_{L P}$ be the LP relaxation bound for the node, $L B$ and $U B$ be the best lower and upper bound obtained in the $B \& B$ algorithm at the time when the node is processed, respectively. Note that if the relaxation is not solved to optimality within a given time limit, the lower bound obtained from solving the relaxation can be used as $l b_{\text {Relax }}$, because a lower bound of a relaxation problem is a valid lower bound of the original problem. If $l b_{\text {Relax }}$ is larger than $U B$, then the node can be pruned. Otherwise, if $l b_{\text {Relax }}$ is greater than $l b_{L P}$, the lower bound of the node is improved. To impose the new lower bound in the B\&B algorithm, we add a cutting plane $c^{T} x \geq l b_{\text {Relax }}$ to the children nodes. However, adding such cutting planes increases the size of the problem to be solved at the children nodes and may have a negative influence on the overall efficiency of the B\&B algorithm. Thus, we consider adding the associated cutting plane only when the improvement on the lower bound is larger than a threshold value $\Delta$, that is when $l b_{\text {Relax }}-l b_{L P}>\Delta$.

Even if the additional relaxations can be solved efficiently, it is not practical to solve them at every node of the $B \& B$ tree. Therefore, we select a subset of the nodes in the B\&B tree to solve the additional relaxations. A pseudo code of the scheme is given in Algorithm 1.

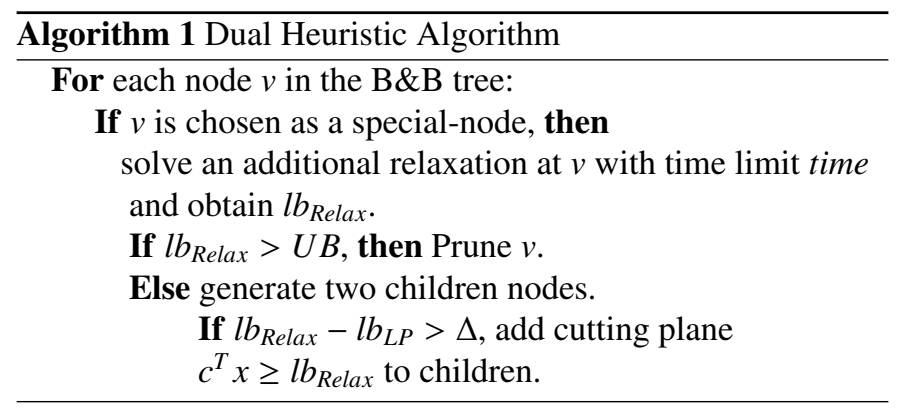

The implementation of the dual heuristic algorithm involves determining which nodes of the B\&B tree should be chosen as special-nodes, and how we should define and solve the relaxations. Specifically, for a chosen special-node, there are several decisions to be made: How should we choose the presentconstraints for the relaxation? How many present-constraints should be used? Which relaxation algorithm should be used for dealing with the relaxed-constraints? As we try to answer these questions in the following sections, the details of the Dual Heuristic Algorithm will be revealed.

\section{Generating Instances}

Since our goal is to solve hard MKPs by improving lower bounds with the dual heuristic algorithm, we focus on instances of large size, especially those with a large number of constraints. We denote the number of unbounded integer variables by $n$, and the number of constraints by $m$. We choose $n \in\{50,100\}$ and $m \in\{100,500,1000\}$. The constraint coefficients $a_{i j}$ are randomly generated integers from the uniform distribution $U(0,1000)$, for $i=1, \ldots, m$ and $j=1, \ldots, n$. 
As many studies indicate (see [5], [6], [7], [8] and [9]), constraint tightness and objective-constraint correlation contribute the most to the hardness of MKP instances. Therefore, we create a test bed composed of instances with different levels of these two measures. Let $K \geq 0$ be a parameter that controls the correlation between objective coefficients and constraint coefficients. Given $K$, the objective coefficients are generated as $c_{j}=\sum_{i=1}^{m} a_{i j} / m+K u$, where $u$ is a random number between zero and one. When $K=0, c_{j}$ is a function of the constraint coefficients in column $j$, for all $j$. Thus the objective function and the constraints are strongly correlated. As $K$ increases, the correlation deteriorates. Let $\alpha \geq 0$ be a parameter controlling the tightness of all constraints. Given $\alpha$, the right-hand side of the constraints are generated as $b_{i}=\left\lceil\alpha \sum_{j=1}^{n} a_{i j}\right\rceil$ for $i=1, \ldots, m$. In general, a large $\alpha$ indicates that the constraints are tight, while a small $\alpha$ means the constraints are loose. Note that we use the same tightness parameter on all constraints, since when the constraints have different levels of tightness, tight constraints are needed to define the convex hull of the feasible region and loose constraints are likely to be "redundant". That is, a feasible solution satisfying the tight constraints is likely to satisfy the loose constraints, and in such a case, the difficulty for solving the instances may be reduced. Since the variables are unbounded, $\alpha$ can be arbitrarily large, but we find that instances with $\alpha>1$ are often easy to solve and not of computational interest. Therefore, we choose the following values for $K \in\{0,100,500,1000\}$ and $\alpha \in\{0.25,0.5,0.75,1\}$.

For each combination of $n, m, \alpha$, and $K$, we generate a group of 10 instances. To test the impact of $\alpha$ on problem hardness, for a fixed combination of $n, m$, and $K$, we use the same constraint matrix and objective function, and only vary $\alpha$ to change the right-hand sides of the constraints. Similarly, to test the impact of $K$, for a fixed combination of $n, m$, and $\alpha$, we use the same constraints and only modify the objective function by varying $K$. Therefore, our initial test set is composed of $2 \times 3 \times 4 \times 4 \times 10=$ 960 total instances in 96 groups.

Finally from the 960 instances, we select a test bed of instances that will be used for the computational experiments in the remainder of the paper. From initial testing we observe that varying $K$ and $\alpha$ has the same impact on results for different instance sizes, hence we choose to focus on instances of size $n=50$ and $m=500$.

Further computational tests show that for small $K$, smaller $\alpha$ leads to harder instances and for large $K$, larger $\alpha$ yields harder instances. From examining the best found solutions, we are able to derive some characteristics of good solutions. For instances with small $K$, most of the variables have value zero or one in the solution, which indicates that there are no columns that are much more important than others. Therefore, when the right-hand side increases, more columns are needed in the solution, which makes the instances easier to solve. However, for large $K$, the solutions have a much smaller number of positive components corresponding to the "important" columns. As $\alpha$ increases, the value of the "important" components in a good solution increases, thus the problem is harder to solve. Therefore, we select two sets of instances for the computational experiments in the rest of the paper, which feature different char- acteristics in constraint tightness and objective-constraint correlation as follows:

- Set I: The set has 10 randomly generated instances with $n=50, m=500, K=0$, and $\alpha=0.25$. This set represents the instances with loose constraints, where the objective and constraint coefficients are strongly correlated.

- Set II: The set has 10 randomly generated instances with $n=50, m=500, K=500$, and $\alpha=1$. This set represents the instances with tight constraints, where the objective and constraint coefficients are weakly correlated.

Set I contains instances where the "importance" of all columns is similar, and thus the problem hardness is caused by the difficulty of choosing the right columns to use in the solution. Set II contains instances where some columns are more important than others, thus the problem hardness is caused by the large right-hand side value of the constraints.

\section{Choosing Present-Constraints}

We first study the effect of different methods for choosing present-constraints to produce good lower bounds using constraint relaxation. For MKP, since all the constraints are knapsack constraints, it is generally hard to determine presentconstraints that will lead to better bounds. Because our primary goal is to find a good way to choose the best presentconstraints to obtain strong lower bounds, computational times are not important in this section and we use a time limit of one hour for tests. Also, we want to focus on the impact of presentconstraints on lower bounds, thus we use relaxations that drop all relaxed-constraints.

We computationally study the impact of using linear relaxation dual variable information to choose present-constraints. Since an optimal linear dual solution can be interpreted as the marginal price of constraints, constraints with larger dual values are more important for solving the LP relaxation. In addition, constraints with small positive slack values in an optimal LP solution may be tight in an IP solution. Therefore, we choose present-constraints first in non-increasing order of the dual values that are nonzero, and then in non-decreasing order of the slack values when the dual solutions are zero.

The lower bounds obtained from relaxations with presentconstraints that are chosen by this order are indicated by "LO" in Table 1 with a varying number of present-constraints. For comparison, we obtain lower bounds from ten different relaxations using the same number of randomly chosen presentconstraints, and report the minimum, maximum, and average of the lower bounds obtained, indicated by "min", "max", and "ave". We present the average results of all ten instances for both $K=0$ and $K=500$.

In order to examine the impact of the number of presentconstraints, in Table 1 we report the lower bounds obtained within 1 hour while varying the number of present-constraints from 40 to 400, which is indicated as \# Present in the first column. If the relaxation problems are not solved to optimality within the time limit, we take the lower bounds on the objective values, since a lower bound of a relaxation problem is also a 
Table 1: Choosing Present Constraints: Average Lower Bounds of All Instances

\begin{tabular}{|c|c|cc|}
\hline \#Present & & $\mathrm{K}=0$ & $\mathrm{~K}=500$ \\
\hline 40 & $\min$ & 6,375 & 30,079 \\
& max & 6,496 & 32,363 \\
& ave & 6,432 & 31,297 \\
& LO & 6,687 & 36,336 \\
\hline 80 & $\min$ & 6,859 & 32,348 \\
& max & 6,943 & 34,066 \\
& ave & 6,906 & 33,334 \\
& LO & 6,921 & 36,533 \\
\hline 100 & min & 6,923 & 32,896 \\
& max & 7,018 & 34,585 \\
& ave & 6,972 & 33,774 \\
& LO & 6,964 & 36,573 \\
\hline 200 & min & 7,134 & 34,529 \\
& max & 7,164 & 35,617 \\
& ave & 7,151 & 35,137 \\
& LO & 7,153 & 36,581 \\
\hline 300 & min & 7,211 & 35,516 \\
& max & 7,234 & 36,145 \\
& ave & 7,223 & 35,868 \\
& LO & 7,220 & 36,547 \\
\hline 400 & min & 7,282 & 36,045 \\
& max & 7,298 & 36,427 \\
& ave & 7,291 & 36,259 \\
& LO & 7,290 & 36,534 \\
\hline All & LB & 7,335 & 36,518 \\
\hline & LP & 6,234 & 35,978 \\
& UB & 7,910 & 36,719 \\
\hline & & & \\
\hline
\end{tabular}

lower bound of the original problem. In addition, we present the lower bounds obtained by solving the original problems within 1 hour by keeping all constraints present in row "All”. Furthermore, we report the linear relaxation bounds using only original problem constraints, indicated by "LP" and the best known upper bounds UB obtained within 10 hours of using CPLEX to solve the original instances.

The results show that with only 40 present-constraints, the lower bounds obtained from relaxations using "LO" order are on average already better than the LP bounds. For instances with low correlation between the objective function and constraints, that is, $K=500$, the lower bounds with "LO" order are always significantly better than the bounds obtained by randomly choosing the present-constraints. For $K=0$, the lower bounds with "LO" order are often better than or close to the maximum of lower bounds when the present-constraints are chosen randomly, which implies that the constraints chosen by "LO" order are relatively more important constraints.

The differences between the maximum and minimum lower bounds obtained with randomly chosen present-constraints decrease when the number of present-constraints increases, indicating that the choice of present-constraints becomes less important as the number of present-constraints increases. For $K=0$, the minimum lower bounds obtained with a larger number of present-constraints are often greater than the maximum lower bounds obtained with fewer present-constraints, which shows that the impact of the number of present-constraints is stronger than the choice of present-constraints.

In addition, for instances with $K=0$, the lower bounds generally increase when the number of present-constraints increases. While for instances with $K=500$, the lower bounds first increase then decrease as the number of present-constraints increases, which indicates that further extending the set of present-constraints does not help to improve the lower bounds. This, of course, is a consequence of the time limit.

Furthermore, when $K=0$, the solutions of the relaxations are always infeasible to the original problems. On the other hand, for $K=500$, the solutions to the relaxations are often feasible to the original problems. This is because for instances with $K=0$, the LP solutions are highly dual degenerate, thus ordering constraints according to dual solutions and slack values may not be an accurate indication of importance. However, for instances with $K=500$, the optimal LP dual solutions and the slack values are not very degenerate, thus the LP information is an accurate indication of constraint importance.

\section{Comparing Lower Bounds of Relaxations}

Next, we examine the lower bounds obtained from several different relaxation techniques. In particularly, we focus on constraint relaxation, surrogate relaxation with a fixed multiplier and lazy relaxation for the rest of the paper. Again, the goal of this section is to choose relaxations to obtain strong bounds, thus computational times are not important and we use a time limit of one hour for all tests in this section.

As suggested by the computational results in Section 4, we choose the same present-constraints for all relaxations, first in non-increasing order of the dual values that are nonzero, and then in non-decreasing order of the slack values when the dual solutions are zero. We compare the lower bounds obtained from various relaxations with the number of present-constraints varying from 40 to 400 . If the relaxations are not solved to optimality within the time limit, we use the lower bounds obtained in solving the relaxations. In further experiments, we observed that even with a time limit of five hours, similar trends are observed. Table 2 displays the average results obtained for all instances in both sets computed in one hour.

In Table 2, row "All" contains the lower bounds obtained from solving the original problems, that is when all 500 constraints are present. In the rest of the table, we display the differences between the lower bounds obtained from solving the relaxations and the bounds in row "All". Therefore, a positive number indicates that the lower bound is improved by using the relaxation. Row "DROP" presents the results from constraint relaxation. The constraint relaxation of MKP is formulated by simply dropping the relaxed-constraints. Thus, the constraint relaxation is also an MKP with $m_{1}$ constraints. Furthermore, we consider surrogate relaxations with two types of multipliers. Row "SUR1" displays the lower bounds obtained from surrogate relaxation, where all surrogate multipliers are fixed to 1, thus the surrogate constraint is formulated as:

$$
\sum_{j} \sum_{i=m_{1}+1}^{m_{1}+m_{2}} a_{i j} x_{j} \geq \sum_{i=m_{1}+1}^{m_{1}+m_{2}} b_{i} .
$$

Row "SUR2" shows the results of surrogate relaxation using a scaled surrogate constraint, that is, $\sum_{j}\left\lceil\sum_{i=m_{1}+1}^{m_{1}+m_{2}} a_{i j} /\left|m_{2}\right|\right\rceil x_{j} \geq$ $\left\lfloor\sum_{i=m_{1}+1}^{m_{1}+m_{2}} b_{i} /\left|m_{2}\right|\right\rfloor$. The surrogate constraints for "SUR2" are weaker than surrogate constraints for "SUR1", but the sizes of the constraint coefficients are scaled down, which could be advantageous. Row "Lazy" includes the lower bounds of the objective function by using the B\&B algorithm with lazyconstraints, that is, the lazy relaxation. 
Table 2: Average Lower Bounds Obtained from Various Relaxations

\begin{tabular}{|c|c|c|c|}
\hline & & $K=0$ & $K=500$ \\
\hline \# Present & All & 7,335 & 36,518 \\
\hline 40 & DROP & -646 & -182 \\
& SUR1 & -647 & -181 \\
& SUR2 & -648 & -181 \\
& Lazy & -16 & 51 \\
\hline 80 & DROP & -412 & 15 \\
& SUR1 & -409 & 21 \\
& SUR2 & -412 & 18 \\
& Lazy & -8 & 47 \\
\hline 100 & DROP & -370 & 55 \\
& SUR1 & -364 & 56 \\
& SUR2 & -365 & 55 \\
& Lazy & -2 & 43 \\
\hline 200 & DROP & -183 & 59 \\
& SUR1 & -180 & 52 \\
& SUR2 & -181 & 50 \\
& Lazy & -8 & 39 \\
\hline 300 & DROP & -117 & 27 \\
& SUR1 & -115 & 31 \\
& SUR2 & -114 & 30 \\
& Lazy & -11 & 28 \\
\hline 400 & DROP & -48 & 15 \\
& SUR1 & -48 & 17 \\
& SUR2 & -51 & 14 \\
& Lazy & -5 & 15 \\
\hline \multirow{7}{*}{30}
\end{tabular}

For $K=0$, except for the lazy relaxation, the lower bounds of all relaxations increase when the number of present-constraints increases. For instances with $K=500$, the lower bounds of all relaxations first increase then decrease when the number of present-constraints increases, which indicates again that for such instances, a large number of present-constraints are not helpful in improving the lower bounds within the time limit. The results confirm that using "LO" order to indicate the importance of constraints is meaningful for instances with $K=500$. However, for instances with $K=0$, because of the degeneracy of the optimal LP solution, the number of present-constraints seems to be more important for obtaining good lower bounds for all relaxation algorithms.

The lower bounds obtained by lazy relaxations are often the best among all relaxations. For instances with $K=500$, using any of the relaxations improves the lower bounds significantly when the number of present-constraints is large. When a small number of present-constraints are used, lazy relaxation shows a strong advantage, which indicates that many relaxedconstraints are actually never violated by solutions to the relaxations, and thus can be eliminated without sacrificing the lower bounds. However, for instances with $K=0$, even using the lazy relaxation does not improve the lower bounds. For such instances, determining good present-constraints can be challenging, therefore the relaxations with a fixed set of presentconstraints may not produce good lower bounds. Hence, it may be more efficient to use the dual heuristic algorithm, which allows solving relaxations with diversified sets of presentconstraints.

\section{Implementing the Dual Heuristic Algorithm}

In the previous sections, we have partially answered the questions of how to choose efficient relaxations and effective present-constraints for the dual heuristic algorithm given in Section 2. In this section, we study and implement strategies that determine at which nodes of the B\&B tree the relaxations should be solved. Specifically, we computationally explore which nodes should be chosen as special-nodes, as well as how should the relaxations be defined and solved in the B\&B tree.

As shown in Section 5, besides the lazy relaxation, the surrogate relaxation which has a surrogate constraint obtained from summing up all relaxed-constraints ("SUR1") finds on average the best lower bounds. Thus we use it in the dual algorithm as our relaxation technique. Therefore, the surrogate relaxation for $I P(v)$ at a B\&B tree node $v$ is obtained by keeping the present-constraints $A_{1} x \geq b_{1}$ and adding surrogate constraint (2).

As suggested in Section 4, present-constraints are selected according to the dual variables and slack values of the LP solutions. Because cutting planes may be added during the B\&B procedure, the linear programming dual solutions for the associated subproblems may not be meaningful. Therefore, we use only the slack values of the constraints to select presentconstraints in the relaxations. In particular, we choose presentconstraints with slack values less than a threshold value, and we denote this threshold by Slack. In addition, we do not include cutting planes that are added during the B\&B procedure in the subproblems, because the focus of our experiments is the importance of the original constraints.

We use several criteria to designate a node as special. Since the formulations solved at a node and its children are not much different, solving the relaxations at these nodes may be unnecessary. Thus, we restrict the distance between two specialnodes in the same subtree to be greater than a threshold value, Depth.

To restrict the time spent on solving relaxations for efficiency purposes, we use a threshold value Check_Percent to indicate the largest total percentage of special-nodes over all processed-nodes allowed in the B\&B tree. When the percentage of special-nodes divided by processed-nodes exceeds Check_Percent, we stop solving relaxations until this percent is lowered by processing more nodes.

Furthermore, let the relative gap of a node be defined as $\frac{|U B-L B|}{U B}$, we then choose special-nodes when the relative gaps fall within a required range. Our initial experiments show that the dual heuristic algorithm obtains better lower bounds when we choose special-nodes in two ranges of relative gaps. The first range only includes special-nodes with small relative gaps, that is, when the relative gaps are less or equal to some small value. Because such nodes are more likely to be pruned by solving the relaxations, the size of the $\mathrm{B} \& \mathrm{~B}$ tree is likely to be reduced. The second range includes special-nodes with large relative gaps, that is, when the relative gaps are greater or equal to some value. Since the lower bounds of the B\&B algorithm are determined by the minimum lower bounds of the remaining nodes in the B\&B tree, improving on the worst lower bounds may help to improve the overall lower bounds. We use Gap to indicate the required range of relative gaps for the specialnodes. In addition, to avoid spending too much time on one node, we use a time limit, Time, for working on each relaxation.

For $K=500$, as shown in Section 5, it suffices to treat the relaxed-constraints as lazy-constraints to significantly increase the lower bounds. Therefore in this section we focus on the 
more challenging $\mathrm{K}=0$ instances. We conduct all experiments on 10 randomly generated instances with 50 variables, 500 constraints, $\alpha=0.25$ and $K=0$. For all tests, we use a time limit of 10 hours overall, Slack $=1$ and Depth $=5$. We set Time $=$ 0.05 or 1 second, since we found in initial computations that extending Time to 2 or 5 seconds gives worse lower bounds. As described in Section 2, to force the lower bounds, we add the associated cutting plane when the improvement on the lower bound is larger than a threshold value $\Delta$. To summarize, we compare the results from default CPLEX with the results from the dual heuristic algorithm using two sets of parameters that produce the most competitive results:

Test 1: Check_Percent $=100 \%$, Time $=1$ second, Gap $=0 \%-2 \%$, $\Delta=1$. Thus for all nodes with relative gaps less than $2 \%$, a relaxation is solved with time limit 1 second, a cutting plane is added to children nodes if the lower bound obtained from solving the relaxation is greater than the LP relaxation value by one.

Test 2: Check_Percent $=2 \%$, Time $=0.05$ second, Gap $=8 \%-100 \%$, $\Delta=0$. Thus, for nodes with relative gaps greater than $8 \%$, if the total percentage of special-nodes is less than $2 \%$, a relaxation is solved with time limit 0.05 second and a cutting plane is added to children nodes if the lower bound obtained from solving the relaxation is greater than the LP relaxation value.

Computationally, we first compare the lower bounds and relative gaps after a certain number of nodes have been processed in the B\&B tree. Let $L B_{D H}$ and $G a p_{D H}$ be the lower bounds and relative gaps obtained by the dual heuristic algorithm and $L B_{C P L E X}$ and $G a p_{C P L E X}$ be the lower bounds and relative gaps obtained by CPLEX with default settings. We report the average value of $\frac{L B_{D H}-L B_{C P L E X}}{L B_{C P L E X}}$ and $\frac{\operatorname{Gap}_{C P L E X}-G a p_{D H}}{\operatorname{Gap}_{C P L E X}}$ of all instances in Figure 1.

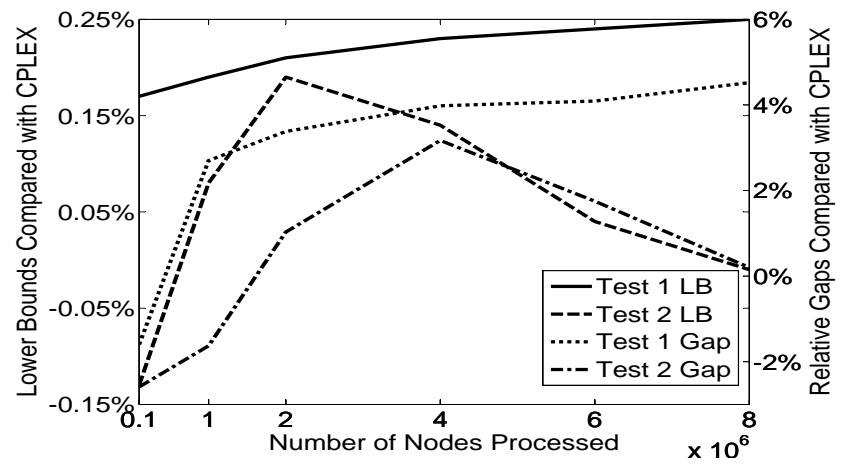

Figure 1: Test 1 and Test 2 Results Compared with CPLEX

Figure 1 shows that the lower bounds are improved in both tests when processing more than 1 million nodes in the $\mathrm{B} \& \mathrm{~B}$ tree. In particular, Test 1 shows the greatest and continuous improvement on the lower bounds over CPLEX, where specialnodes are chosen to have small relative gaps. For Test 2, the improvements are usually observed in the earlier stages of the B\&B algorithm, since special-nodes are chosen to be those with large relative gaps that appear in the early stages. When the percentage of special-nodes decreases as the relative gap falls, the improvement on lower bounds also decreases. We also observe that over $99 \%$ of the special-nodes are pruned by solving the relaxations in Test 1 for all instances. However, nodes are rarely pruned in Test 2. Although the nodes pruned in Test 1 are the ones with small relative gaps, the lower bounds can still be improved. The relative gaps are also improved in both tests. Test 1 shows an improvement of at least $2 \%$ compared with CPLEX, and the advantage extends when more nodes are processed.

When analyzing the run-time efficiency of the dual algorithm, we compare the lower bounds and relative gaps over time and report the average results over all instances in Figure 2. Because of the fact that our algorithm invokes callback-handling in CPLEX at each node of the B\&B tree, we account for the callback time to get a fair comparison with CPLEX by using a dummy callback in CPLEX runs. This approach to obtaining a fair comparison was suggested in [10].

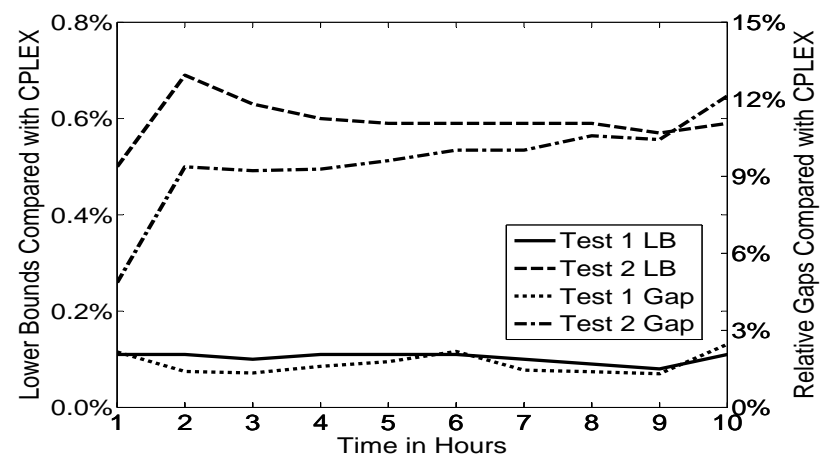

Figure 2: Test 1 and Test 2 Results Compared with CPLEX with Adjusted Time

As Figure 2 shows that the dual heuristic algorithm performs better in Test 2 than in Test 1, since it spends a shorter time per node on average in the $B \& B$ tree due to the relatively low percentage of special-nodes in Test 2 . The dual heuristic in both tests outperforms CPLEX, and the relative gaps obtained in Test 2 within the same adjusted time is improved by up to $12 \%$, an improvement that could take hours to achieve by using CPLEX with default settings.

Our results indicate that the dual heuristic algorithm can be effective in improving lower bounds for the B\&B algorithm for properly chosen parameters. In an extended computational study we considered dynamically modifying parameters Check_Percent, Gap and Slack to balance the time efficiency and the effectiveness of the dual heuristic algorithm. However, the results did not show a significant improvement over the results obtained for Test 1 and Test 2. Thus we do not report the results here.

In addition to the knapsack instances, we tested our algorithm on a few instances from MIPLIB with the number of constraints larger than the number of variables. Among these instances, instance pw-myciel4 is solved within 30 minutes using the dual heuristic algorithm, compared to 65 minutes using default CPLEX. In addition, we report results on instance reblock354 in Table 3, where $L B_{-} I m p r$ is $\frac{L B_{D H}-L B_{C P L E X}}{L B_{C P L E X}}$ and Gap_Impr is 
$\frac{\operatorname{Gap}_{C P L E X}-\text { Gap }_{D H}}{\text { Gap }_{C P L E X}}$. The results on this instance suggests that the dual algorithm, possibly with some modifications, appears to be promising for general MIPs.

\begin{tabular}{|c|ccccc|}
\multicolumn{7}{c}{ Table 3: Results on reblock354 } \\
\hline Hour & 2 & 4 & 6 & 8 & 10 \\
\hline LB_Impr & $0.15 \%$ & $0.14 \%$ & $0.14 \%$ & $0.14 \%$ & $0.14 \%$ \\
\hline Gap_Impr & $40.00 \%$ & $39.47 \%$ & $38.89 \%$ & $41.67 \%$ & $40.63 \%$ \\
\hline
\end{tabular}

\section{Conclusions and future research}

We studied relaxation algorithms to obtain lower bounds for MKP, and examined the impact of the number of presentconstraints on the lower bounds obtained from the relaxations. Our results indicated that for MKP, the quality of lower bounds depends heavily on the choice and the number of presentconstraints.

In addition, we developed a dual heuristic algorithm that solves relaxations during the execution of the $\mathrm{B} \& \mathrm{~B}$ algorithm with various present-constraints for improving lower bounds. The results indicated that by carefully choosing special-nodes, the quality of the lower bounds and relative gaps can be improved significantly. On the other hand, despite the time spent on callbacks, the efficiency of the dual heuristic algorithm is closely related to the efficiency of solving the relaxation subproblems, and in this we see two potential directions for future work.

Exploring a diversified neighborhood to find better feasible solutions has been considered in some effective primal heuristics, a similar idea for the dual heuristic is to limit the number of the same present-constraints that are used in two special-nodes. Specifically, let $v$ be a special-node where the lower bound is not improved by solving the relaxation. Then for special-nodes that are in the subtree rooted at $v$, we may require the use of a somewhat different set of present-constraints. This strategy reduces the dependency of our algorithm on the linear relaxation solutions, and thus may lead to good solutions when the LPs are highly degenerate.

Furthermore, for almost all special-nodes, the number of tight constraints included in the relaxation was less than 25 in our experiments. This indicates that with only a small number of present-constraints, the lower bounds of the nodes can still be improved by solving the relaxations. This provides us hope of improving the efficiency of the algorithm by using a single surrogate constraint in relaxations, where only a small number of constraints have large weights. That is, we can potentially obtain lower bounds by solving $\max \left\{c^{T} x: \lambda A x \geq \lambda b, x \in \mathbb{Z}_{+}^{n}\right\}$. The weights of the constraints, indicated by the vector $\lambda$, can be updated using information obtained during the B\&B algorithm, and the weights can be made larger for tight constraints at the special-nodes. Since for most special-nodes the number of tight constraints is usually small, the quality of the lower bounds obtained with the single surrogate constraint may not be sacrificed much, but the efficiency in solving the relaxation as a knapsack problem may be improved. Finally we note that our approach is suitable for a parallel implementation since parallelism gives the opportunity to use a wide variety of present-constraints and parameters and then choose the best solution found among all of the solvers.

[1] A. Lodi, Mixed integer programming computation, Springer, 2010.

[2] M. Fischetti, F. Glover, A. Lodi, The feasibility pump, Mathematical Programming 104 (1) (2005) 91-104.

[3] M. Fischetti, A. Lodi, Local branching, Mathematical Programming 98 (1) (2003) 23-47.

[4] E. Danna, E. Rothberg, C. Pape, Exploring relaxation induced neighborhoods to improve MIP solutions, Mathematical Programming 102 (1) (2005) 71-90.

[5] S. Martello, P. Toth, Knapsack problems: algorithms and computer implementations, John Wiley \& Sons, Inc., 1990.

[6] J. Beasley, OR - library: Distributing test problems by electronic mail, Journal of the Operational Research Society 41 (11) (1990) 1069-1072.

[7] A. Freville, G. Plateau, Hard 0-1 multiknapsack test problems for size reduction methods, Investigation Operativa 1 (1990) 251-270.

[8] P. Chu, J. Beasley, A genetic algorithm for the multidimensional knapsack problem, Journal of Heuristics 4 (1) (1998) 63-86.

[9] M. Osorio, F. Glover, P. Hammer, Cutting and surrogate constraint analysis for improved multidimensional knapsack solutions, Annals of Operations Research 117 (1) (2002) 71-93.

[10] R. Carvajal, S. Ahmed, G. Nemhauser, K. Furman, V. Goel, Y. Shao, Using diversification, communication and parallelism to solve mixed-integer linear programs, Operations Research Letters 42 (2) (2014) 186-189.

\section{Acknowledgements}

This work was supported in part by the Air Force Office of Scientific Research and the National Science Foundation.

We also wish to thank an anonymous referee whose comments helped us to improve the clarity of the paper. 